\title{
An enigmatic long-lasting $\gamma$-ray burst not accompanied by a bright supernova
}

\begin{abstract}
M. Della Valle ${ }^{1}$, G. Chincarini ${ }^{2,3}$, N. Panagia ${ }^{4,5,6}$, G. Tagliaferri $^{3}$, D. Malesani ${ }^{7}$, V. Testa ${ }^{8}$, D. Fugazza ${ }^{3,2}$, S. Campana ${ }^{3}$, S. Covino ${ }^{3}$, V. Mangano ${ }^{9}$, L.A. Antonelli $^{8,10}$, P. D'Avanzo ${ }^{3,11}$, K. Hurley ${ }^{12}$, I. F. Mirabel ${ }^{13}$, L. J. Pellizza ${ }^{14}$,
\end{abstract} S. Piranomonte ${ }^{8} \&$ L. Stella ${ }^{8}$

${ }^{1}$ INAF, Osservatorio Astrofisico di Arcetri, largo E. Fermi 5, I-50125 Firenze, Italy.

${ }^{2}$ Dipartimento di Fisica, Università degli Studi di Milano-Bicocca, piazza delle Scienze 3, I-20126 Milano, Italy.

${ }^{3}$ INAF, Osservatorio Astronomico di Brera, via E. Bianchi 46, I-23807 Merate (Lc), Italy.

${ }^{4}$ Space Telescope Science Institute, 3700 San Martin Drive, Baltimore, Maryland 21218 Baltimore, USA.

${ }^{5}$ Istituto Nazionale di Astrofisica, viale del Parco Mellini 84, I-00136 Roma, Italy.

${ }^{6}$ Supernova Ltd., Olde Yord Village \#131, Northsound Road, Virgin Gorda, British Virgin Islands.

${ }^{7}$ International School for Advanced Studies (SISSA/ISAS), via Beirut 2-4, I-34014 Trieste, Italy.

${ }^{8}$ INAF, Osservatorio Astronomico di Roma, via di Frascati 33, I-00040 Monteporzio Catone (Roma), Italy.

${ }^{9}$ INAF, Istituto di Astrofisica Spaziale e Fisica Cosmica di Palermo, via U. La Malfa 153, I-90146 Palermo, Italy.

${ }^{10}$ ASI Science Data Center, via G. Galilei, I-00044 Frascati, Italy.

${ }^{11}$ Dipartimento di Fisica e Matematica, Università dell'Insubria, via Valleggio 11, I-22100 Como, Italy.

${ }^{12}$ University of California, Berkeley, Space Sciences Laboratory, Berkeley, California 94720-7450, USA.

${ }^{13}$ European Southern Observatory, Alonso de Córdova 3107, Vitacura, Casilla 19001, Santiago 19, Chile.

${ }^{14}$ Instituto de Astronomía y Física del Espacio (CONICET/UBA), Casilla de Correos 67 Suc. 28, (1428) Buenos Aires, Argentina. 
Gamma-ray bursts (GRBs) are short, intense flashes of soft $\gamma$-rays coming from the distant Universe. Long-duration GRBs (those lasting more than $\sim 2 \mathrm{~s}$ ) are believed to originate from the deaths of massive stars $^{1}$, mainly on the basis of a handful of solid associations between GRBs and supernovae ${ }^{2-7}$. GRB 060614, one of the closest GRBs discovered, consisted of a 5-s hard spike followed by softer, brighter emission that lasted for $\sim 100 \mathrm{~s}$ (refs 8, 9). Here we report deep optical observations of GRB 060614 showing no emerging supernova with absolute visual magnitude brighter than $M_{\mathrm{V}}=-13.7$. Any supernova associated with GRB 060614 was therefore at least 100 times fainter, at optical wavelengths, than the other supernovae associated with GRBs ${ }^{10}$. This demonstrates that some long-lasting GRBs can either be associated with a very faint supernova or produced by different phenomena.

Following the discovery of the X-ray and optical afterglow of GRB $060614^{9}$, we observed it with the European Southern Observatory (ESO) $8.2 \mathrm{~m}$ Very Large Telescope (VLT). The spectrum of the host galaxy exhibits nebular emission lines (Fig. 1), which reveal ongoing star formation and the presence of young, massive stars. The specific star formation rate, normalized to the host luminosity (B-band magnitude $M_{\mathrm{B}}=-15.5$ ), is about $2 M_{\odot} \mathrm{yr}^{-1} L_{*}^{-1}$, where $M_{\odot}$ is the solar mass and $L_{*}$ is the typical luminosity of field galaxies. This value is comparable to that exhibited by the Milky Way, and is at the low end of the distribution found for long-duration GRB hosts ${ }^{11}$. The galaxy is also fainter than most GRB hosts ${ }^{12}$.

Starting $15 \mathrm{~h}$ after the burst, we monitored the light curve of the optical transient associated with GRB 060614. Deep observations obtained in the R band (roughly corresponding to the $\mathrm{V}$ band in the GRB rest frame) up to $65 \mathrm{~d}$ after the burst did not reveal the emergence of a supernova component (Fig. 2; see also refs 13,14); such a component has been often observed in a number of nearby long-duration GRBs. Our data are consistent with no supernova contribution (blue line). Adopting as a template the light curve of SN $1998 \mathrm{bw}^{2}$, we constrain the brightness of a supernova coincident with GRB 060614 to be at least 5.6 mag fainter than the template ( $3 \sigma$ limit; green lines in Fig. 2). This corresponds to a peak absolute magnitude $M_{\mathrm{V}}>-13.5$. Similar limiting magnitudes are obtained adopting different supernova light curve shapes. A brighter supernova (yellow lines in Fig. 2) would provide a totally inadequate fit. The faintness of a possible supernova at optical 
wavelengths was further confirmed by a series of about ten spectra obtained at the VLT in the 4,500-8,000 $\AA$ wavelenght range, between 2006 June 15 and 2006 July 30. None of them shows the broad undulations due to the very high expansion velocities $\left(\sim 30,000 \mathrm{~km} \mathrm{~s}^{-1}\right)$ typical of the supernovae ${ }^{15}$ associated with GRBs.

Such faintness cannot be due to dust extinction. First, the afterglow optical spectra are not particularly red (from our BVRIJK photometry we measure a spectral index $\beta=0.94$ at $\sim 1.7 \mathrm{~d}$ after the GRB). The afterglow is also bright in the ultraviolet ${ }^{9}$, where extinction would be more severe. X-ray spectra (V.M. et al., manuscript in preparation) also show little absorbing material along the line of sight (yielding a rest-frame hydrogen column density $N_{\mathrm{H}}<2 \times 10^{20} \mathrm{~cm}^{-2}$ at $90 \%$ confidence level). Furthermore, by modelling the broad-band optical/X-ray spectral energy distribution (see Supplementary Fig. 1), we can estimate that the source was affected by less than 0.2 mag of extinction in the observed $R$ band. Allowing for this amount of extinction, we can refine our limit for the supernova peak magnitude to $M_{\mathrm{V}}>-13.7$.

So far only type-Ib/c events have been clearly associated with $\mathrm{GRBs}^{1}$ and, had the progenitor of GRB 060614 been one of them, its expected colour at maximum light would be $B-V \approx 0.5 \pm 0.1$, as measured in well-observed events such as SN 2006aj ${ }^{10}$, SN 2002ap ${ }^{16}$ and SN 1998bw ${ }^{2}$, as well in several other type-Ib/c SNe. From the tables of ref. 17, we find that this colour corresponds to an effective temperature $T \approx 6,500 \mathrm{~K}$, which, combined with the lower limit to the absolute magnitude, provides a strict upper limit to the bolometric luminosity, at maximum light, of $L<10^{41} \mathrm{erg} \mathrm{s}^{-1}$. Therefore the radius of the emitting region is constrained to be $R \approx \sqrt{L /\left(4 \pi \sigma T^{4}\right)}<$ $2.8 \times 10^{14} \mathrm{~cm}$ (where $\sigma$ is the Stefan-Boltzmann constant). As the rise times to maximum light of type-Ib/c supernovae range between 10 and $20 \mathrm{~d}$ (refs 10, 18-20) in the $\mathrm{V}$ band, the upper limit to the radius implies an upper limit to the expansion velocity in the range $1,600-3,200 \mathrm{~km} \mathrm{~s}^{-1}$. This value is an order of magnitude smaller than that observed in SNe associated with $\mathrm{GRBs}^{7,15}$.

The low expansion velocity and the faint luminosity implied for a possible supernova progenitor are reminiscent of a class of very faint core-collapse supernovae recently discovered ${ }^{21}$ in the local Universe. They are of type II, have absolute magnitudes at maximum in the range $-13>M_{\mathrm{V}}>-15$, and show very small expansion velocities $\left(\sim 1,000 \mathrm{~km} \mathrm{~s}^{-1}\right)$. The properties of such objects may well be consistent with the available data on GRB 060614 . 
Faint type-II SNe have been interpreted in terms of the collapse of massive stars with an explosion energy so small that most of ${ }^{56} \mathrm{Ni}$ falls back onto the compact stellar remnant ${ }^{22}$. Such supernovae share properties with the present case, both in terms of observational characteristics and because they are expected to give rise to a black hole (which is believed to be necessary for the production of a GRB). However, the possibility that such supernova progenitors are able to produce GRBs has yet to be explored. In particular, the stellar envelope would need to be absent for the relativistic jets to emerge out of the star. GRB 060614 might thus be an example of a fallback supernova of type $\mathrm{Ib} / \mathrm{c}$. The small amount of nickel $\left(<10^{-3} M_{\odot}\right)$, responsible for the very low luminosity, might possibly also provide little heating to the ejecta, leading to an unusually low temperature $(T \approx 2,000 \mathrm{~K})$ and allowing for larger velocities (as $v \propto T^{-2}$ ). In any case, GRB 060614 may be the prototype of a new class of GRBs originating from a new kind of massive stars death, different from those producing both classical long-duration (associated with bright type-Ib/c supernovae) and short-duration (possibly originating in binary system mergers ${ }^{23}$ ) GRBs. Some evidence for this idea comes from the high-energy properties of this GRB, which contemporaneously exhibits features typical of both the long and short GRB classes ${ }^{8}$. Indeed, scenarios in which the GRB was not directly connected to a supernova explosion ${ }^{24}$ cannot be excluded by our data (though they are not required). For example, our data would be compatible with a supernova exploding before the $\mathrm{GRB}^{25}$. Also, a binary merger mechanism ${ }^{26}$, similar to that proposed to power short-duration GRBs, or some type of collapsar model ${ }^{27}$, are not expected to produce a supernova. These results challenge the commonly accepted scenario, in which long-duration GRBs are produced only together with very bright supernova explosions. Not all GRBs are produced in such a way.

1. Woosley, S. E. \& Bloom, J. S. The supernova-gamma-ray burst connection. Annu. Rev. Astron. Astrophys. 44, 507-556 (2006).

2. Galama, T. J. et al. An unusual supernova in the error box of the $\gamma$-ray burst of 25 April 1998. Nature 395, 670-672 (1998).

3. Stanek, K. Z. et al. Spectroscopic discovery of the supernova 2003dh associated with GRB 030329. Astrophys. J. 591, L17-L20 (2003). 
4. Hjorth, J. et al. A very energetic supernova associated with the $\gamma$-ray burst of 29 March 2003. Nature 423, 847-850 (2003).

5. Malesani, D. et al. SN 2003lw and GRB 031203: A bright supernova for a faint gamma-ray burst. Astrophys. J. 609, L5-L8 (2004).

6. Pian, E. et al. An optical supernova associated with the X-ray flash XRF 060218. Nature 442, 1011-1013 (2006).

7. Campana, S. et al. The association of GRB 060218 with a supernova and the evolution of the shock wave. Nature 442, 1008-1010 (2006).

8. Gehrels, N. et al. A new $\gamma$-ray burst classification scheme from GRB 060614. Nature, 444, 1044-1046 (2006).

9. Parsons, A. M. et al. GRB 060614: Swift detection of a burst with a bright optical and X-ray counterpart. GCN Circ. 5252 (2006).

10. Ferrero, P. et al. The GRB060218/SN 2006aj event in the context of other gamma-ray burst supernovae. Astron. Astrophys. 457, 857-864 (2006).

11. Christensen, L., Hjorth, J. \& Gorosabel, J. UV star-formation rates of GRB host galaxies. Astron. Astrophis. 425, 913-926 (2004).

12. Fruchter, A. S. et al. Long $\gamma$-ray bursts and core-collapse supernovae have different environments. Nature, 441, 463-468 (2006).

13. Gal-Yam, A. et al. A novel explosive process is required for the $\gamma$-ray burst GRB 060614. Nature, 444, 1053-1055 (2006).

14. Fynbo, J. P. U. et al. No supernovae associated with two long-duration $\gamma$-ray bursts. Nature, 444 1047-1049 (2006).

15. Patat, F. et al. The metamorphosis of SN 1998bw. Astrophys. J., 555, 900-917 (2001).

16. Foley, R. et al. Optical photometry and spectroscopy of the SN 1998bwlike type Ic supernova 2002ap. Publ. Astron. Soc. Pacif. 115, 12201235 (2003). 
17. Romaniello, M., Panagia, N., Scuderi, S. \& Kirshner, R. P. Accurate stellar population studies from multiband photometric observations. Astronom. J. 123, 915-940 (2002).

18. Della Valle, M. et al. Hypernova signatures in the late rebrightening of GRB 050525A. Astrophys. J. 642, L103-L106 (2006).

19. Hamuy, M. Observed and physical properties of core-collapse supernovae. Astrophys. J. 582, 905-914 (2003).

20. Panagia, N. in Supernovae and Gamma-Ray Bursters (ed. Weiler, K. W.) 113-144 (Lecture Notes in Physics, Vol. 598, Springer, Berlin, 2003).

21. Pastorello A. et al. Low luminosity type II supernovae: Spectroscopic and photometric evolution. Mon. Not. R. Astron. Soc. 347, 74-94 (2004).

22. Nomoto, K. et al. in Stellar Collapse (ed. Fryer, C.) 277-325 (Astrophysics and Space Science Library, Vol. 302, Kluwer Academic, Dordrecht, 2004).

23. Eichler, D., Livio, M., Piran, T. \& Schramm, D. N. Nucleosynthesis, neutrino bursts and $\gamma$-rays from coalescing neutron stars. Nature, $\mathbf{3 4 0}$, 126-128 (1989).

24. Blinnikov, S.I. \& Postnov, K.A. A mini-SN model for optical afterglow of GRBs. Mon. Not. R. Astron. Soc. 293, L29-L32 (1998).

25. Vietri, M. \& Stella, L. A gamma-ray burst model with small baryon contamination. Astrophis. J. 507, L45-L48 (1998).

26. Belczynski, K., Bulik, T. \& Rudak, B. Study of gamma-ray burst binary progenitors. Astrophys. J. 571, 394-412 (2002).

27. Woosley, S.E., Zhang, W. \& Heger, A. in Gamma-Ray Burst and Afterglow Astronomy 2001: A Workshop Celebrating the First Year of the HETE Mission (eds Ricker, G. R. \& Vanderspek, R.) 185-192 (AIP Conf. Proc., Vol. 662, American Institute of Physics, New York, 2003).

28. Price, P. A., Berger, E. \& Fox, D. B. GRB 060614: Redshift. GCN Circ. 5275 (2006). 
29. French, F., Melady, G., Hanlon, L., Jelínek, M. \& Kubánek, P. GRB060614: Watcher observation GCN Circ. 5257 (2006).

Supplementary information is linked to the online version of the paper at www. nature.com/nature.

Acknowledgments. This work is based on data collected at the Very Large Telescope operated by the European Southern Observatory. We acknowledge support from the observing staff. This research is supported in Italy by ASI, MIUR-PRIN and INAF-PRIN grants.

Author information. Reprint and permissions information is available at www.nature.com/reprints. The authors declare no competing financial interests. Correspondence and requests for materials should be addressed to M.D.V. (massimo@arcetri.astro.it). 


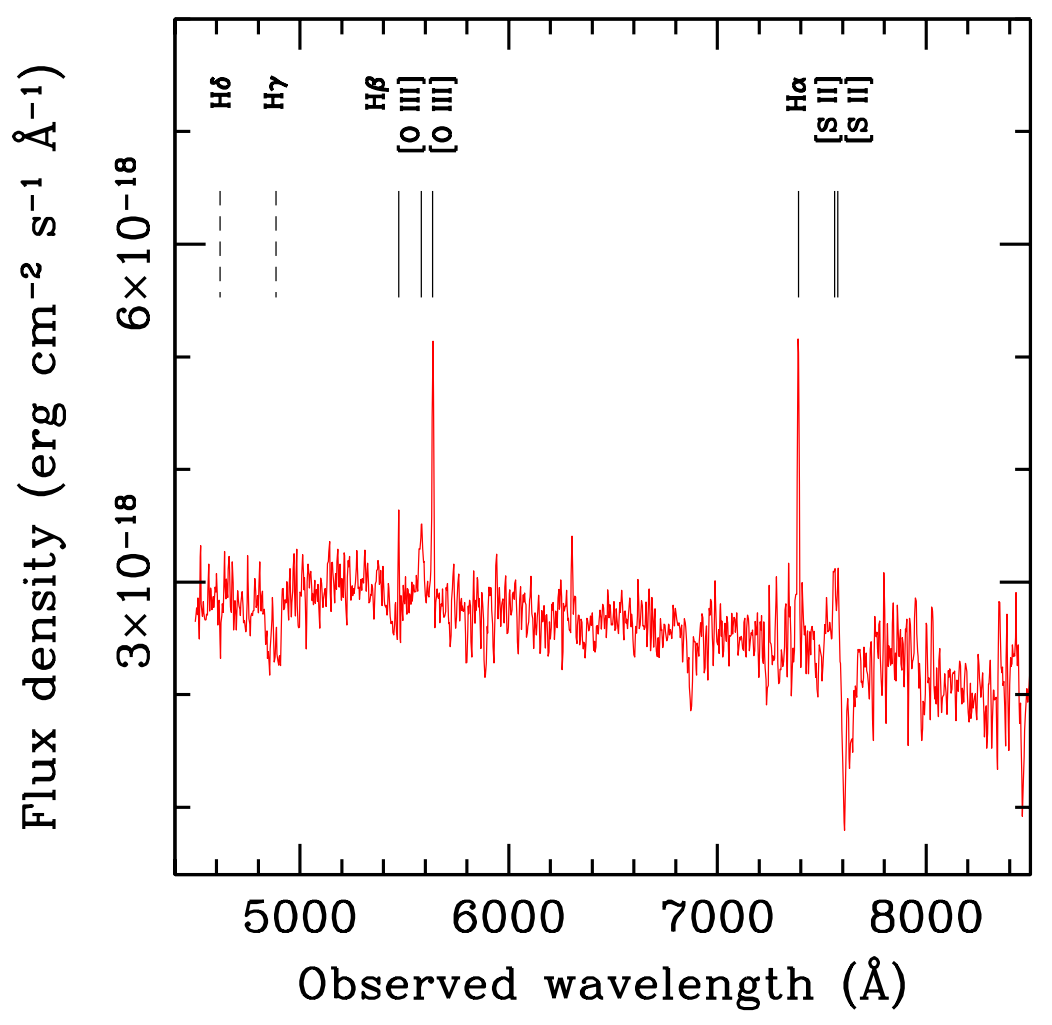

Figure 1 Spectrum of the host galaxy of GRB 060614. This is the average of several observations taken with the VLT equipped with the FORS 2 spectrograph in the period 2006 June 20 to July 30. From the emission features (marked with solid bars) we infer $z=0.1254 \pm 0.0005$. This confirms the redshift proposed in ref. 28. $\mathrm{H} \gamma$ and $\mathrm{H} \delta$ are seen in absorption (dashed bars). The flux from the $\mathrm{H} \alpha$ line, not corrected for internal extinction, amounts to $4.1 \times 10^{-17} \mathrm{erg} \mathrm{cm}^{-2} \mathrm{~s}^{-1}$ (corrected for slit loss). This corresponds to an unobscured star formation rate of $1.3 \times 10^{-2} M_{\odot} \mathrm{yr}^{-1}$. Given the faintness of the galaxy $\left(M_{\mathrm{B}} \approx-15.5\right)$, however, the specific star formation rate $\left(2 M_{\odot} \mathrm{yr}^{-1} L_{*}^{-1}\right.$, assuming for the absolute B-band magnitude of field galaxies $M_{B}^{*}=-21$ ), is not negligible. From the observed flux of the [O III] lines and the limits on [N II], we infer a metallicity larger than $\sim 1 / 20$ solar. 


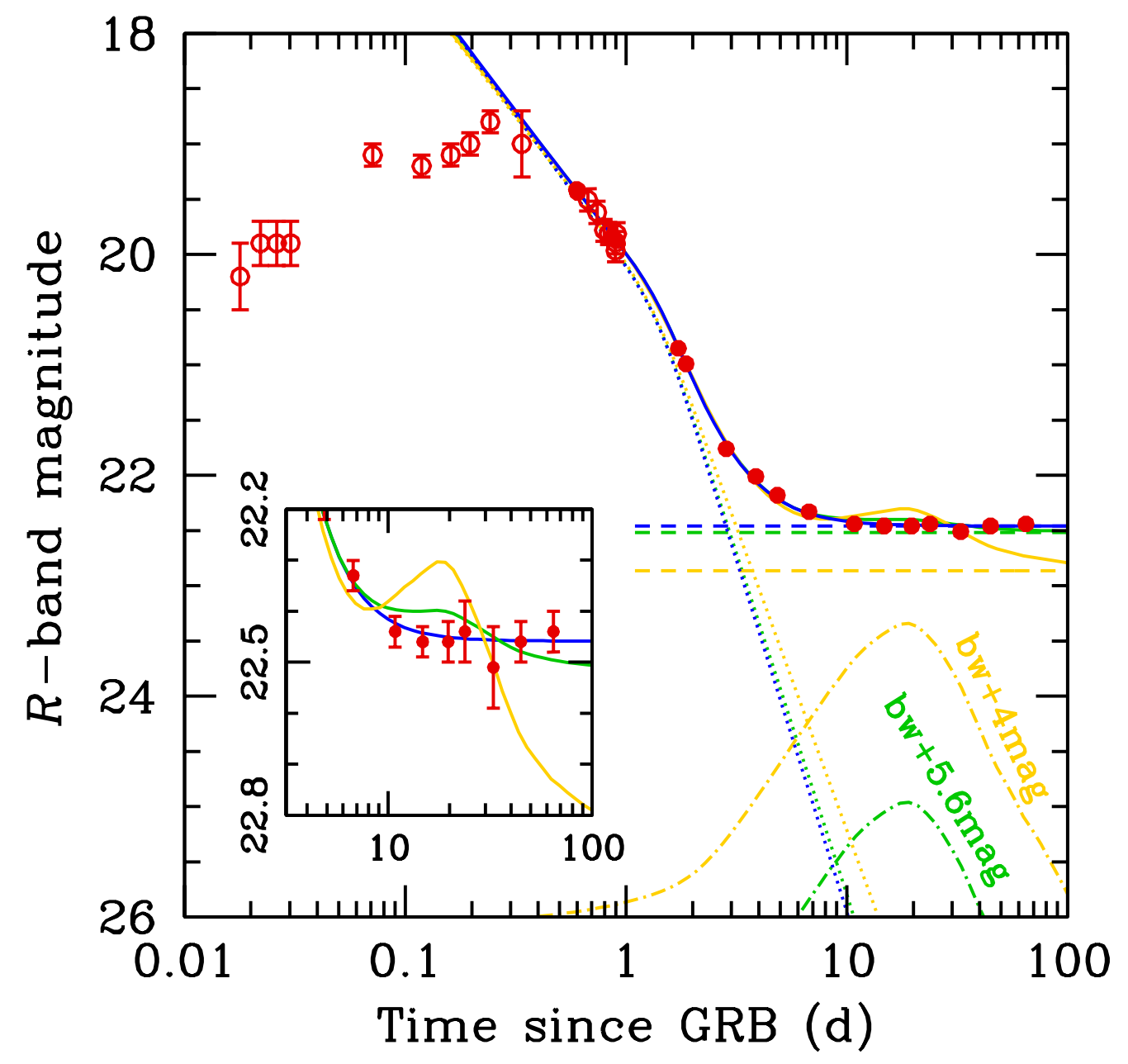

Figure 2 R-band light curve of the GRB 060614 afterglow. Red open circles show data from the literature ${ }^{13,14,29}$ (not used in the fits); red filled circles represent our VLT data (see Supplementary Table 1). Error bars (smaller than symbols for most of our data) show the $1 \sigma$ errors. Photometry was performed adopting large apertures in order to include all the flux from the host galaxy. Flux calibration was achieved by observing several Landolt standard fields. The data were modelled as the sum (solid lines) of three components: the afterglow (dotted lines), the host (dashed lines) and a supernova akin to SN 1998bw but rescaled in flux ('bw'; dot-dashed lines). The different colours correspond to different contributions from the 
supernova: no contribution (blue), a supernova fainter by 5.6 mag (green), and a supernova fainter by 4 mag (yellow). The model shown by gren lines corresponds to the brightest supernova allowed by our data, $M_{\mathrm{V}}>-13.5$, at the $3 \sigma$ level. At the $2 \sigma$ level, the limit is $M_{\mathrm{V}}>-12.9$. The model shown by the yellow lines is clearly inadequate. The inset shows an expanded version of the plot around the peak of a putative supernova. The afterglow component is described by a broken power law with decay indices $\alpha_{1}=1.08 \pm 0.03$ and $\alpha_{2}=2.48 \pm 0.07$, respectively before and after the break at $t_{\text {break }}=1.39 \pm 0.04$ $\mathrm{d}\left(\chi^{2} /\right.$ d.o.f $\left.=15.5 / 20\right)$. Interpreting this as a jet break, the inferred jet halfopening angle is $\vartheta \approx 12^{\circ}$. 


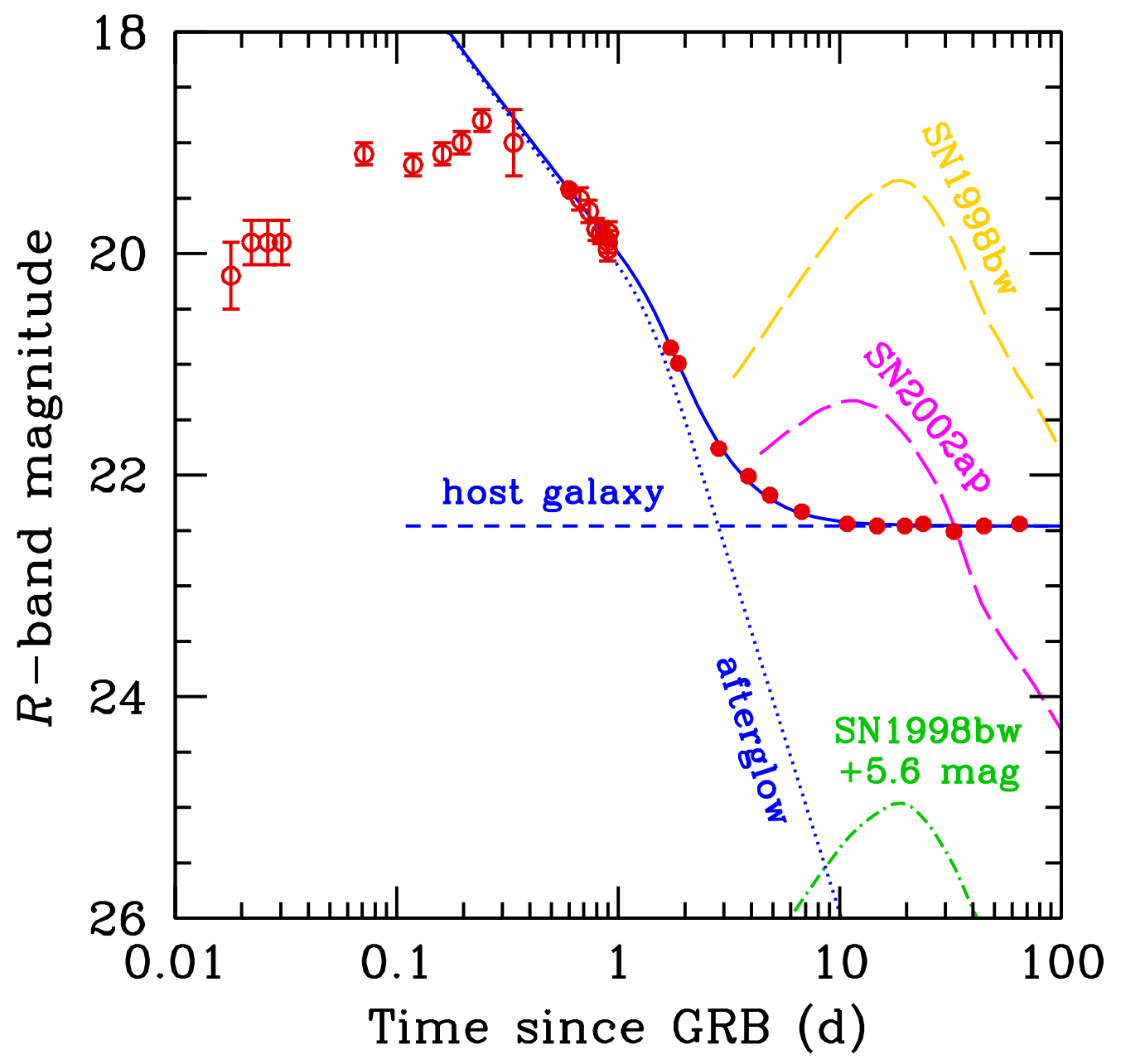

Extra figure | Comparison of the light curve of the GRB 060614 afterglow and those of SN 1998bw and SN 2006aj. The yellow and magenta long-dashed lines show the light curves of two supernovae previously found to be associated with other GRBs reported at $z=0.1254$. The green dot-dashed line shows the brightest supernova allowed by our data at the $3 \sigma$ level. The contribution of the host galaxy (short-dashed line) and of the afterglow (dotted line) are also indicated. The data are the same as in Fig. 2. 


\section{Supplementary material for "An enigmatic long-lasting $\gamma$-ray burst not accompanied by a bright supernova".}

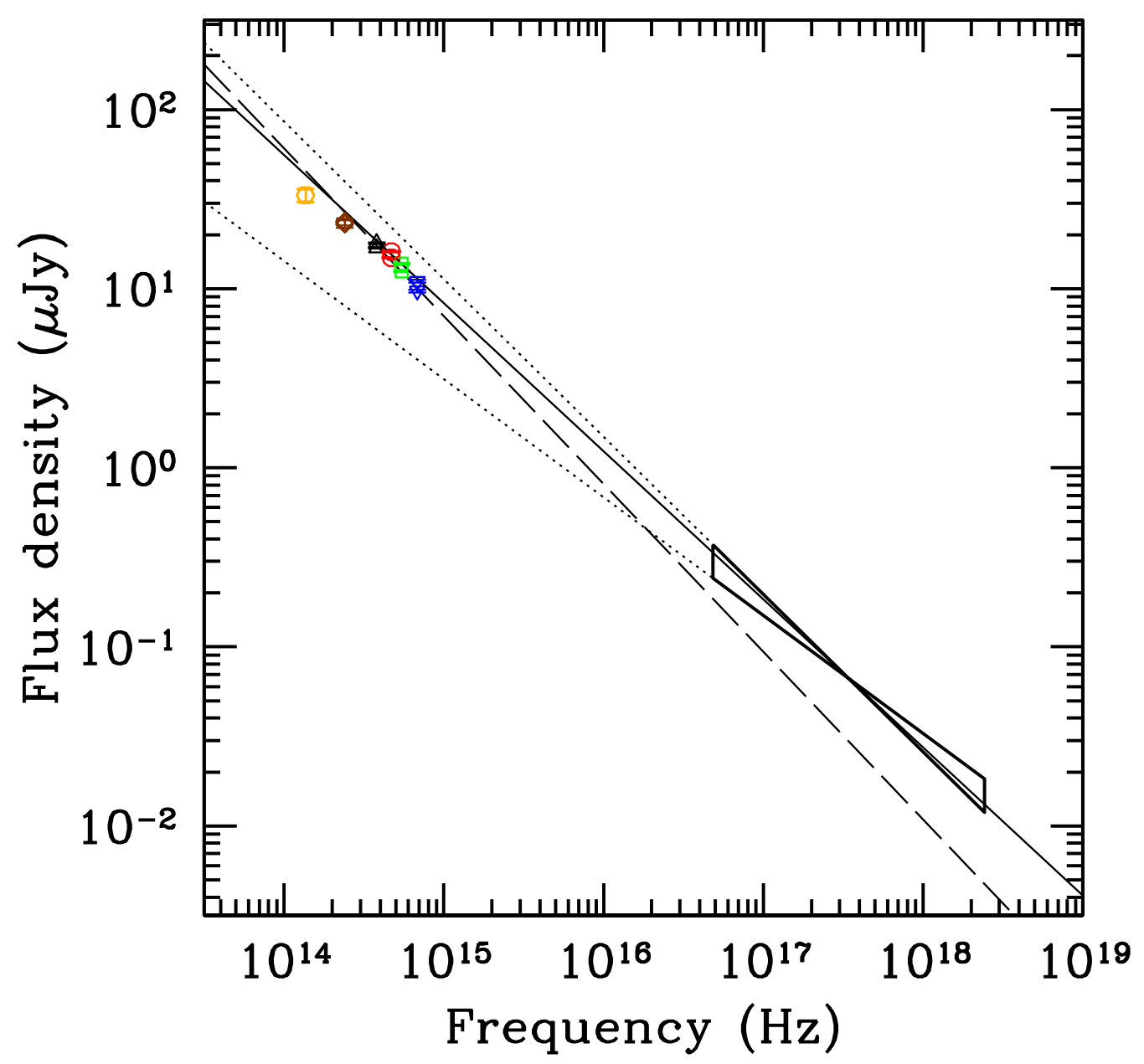

Supplementary Figure 1 | Estimate of the reddening correction. We interpret the afterglow radiation as due to synchrotron emission, as usually assumed for GRB afterglows. In order to build the optical spectral energy distribution (SED), we adopt our BVRIJK photometry and take the X-ray data from the work by V.M. et al. (in preparation). Using our best-fit 
light curve we report all data at the common epoch $t=1.73$ days after the burst (the time around which our measurements cluster). After correcting for the Galactic extinction $\left(A_{V}=0.07 \mathrm{mag}\right)$, the optical spectrum is well fit by a power law $\left(F_{\nu} \propto \nu^{-\beta}\right)$ with index $\beta=0.94 \pm 0.08$ (dashed line). At the same time, the X-ray spectrum is described by a power law with index $\beta=0.77 \pm 0.11$. Supplementary Figure 1 shows the broad-band SED at this epoch. As can be seen, the optical and X-ray data belong to the same powerlaw segment. The common origin of the two components is also confirmed by their similar temporal properties. Assuming an SMC-like extinction curve, to make the optical data match exactly the X-ray flux, an amount of extinction $A_{V}=0.08 \mathrm{mag}$ is required. Since the rest-frame $V$ band corresponds to the $R$ band, this is also the amount of extinction suffered by our measurements. Assuming different extinction curves, the absorption corresponding to the observed $R$ band does not change significantly and is always less than 0.1 mag. 
Supplementary Table 1 Log of observations. The time $t_{0}$ indicates the GRB trigger time, 2006 June 14.53042 UT. Errors are given at the 1- $\sigma$ confidence level.

\begin{tabular}{|c|c|c|c|c|c|c|c|}
\hline $\begin{array}{l}\begin{array}{l}\text { Mean date } \\
(\mathrm{UT})\end{array} \\
\end{array}$ & $\begin{array}{l}t-t_{0} \\
(\text { days })\end{array}$ & Filter & $\begin{array}{l}\text { Exposure } \\
\text { (s) }\end{array}$ & Airmass & $\begin{array}{l}\text { Seeing } \\
\left({ }^{\prime \prime}\right)\end{array}$ & Instrument & Magnitude \\
\hline 2006 Jun 16.27492 & 1.74450 & $\bar{B}$ & $4 \times 90$ & 1.23 & 0.60 & "VLT+FORS1 & $21.72 \pm 0.02$ \\
\hline 2006 Sep 02.05099 & 79.52057 & $B$ & $10 \times 120$ & 1.25 & 1.30 & VLT+FORS1 & $23.62 \pm 0.15$ \\
\hline 2006 Jun 16.25076 & 1.72034 & $V$ & $2 \times 120$ & 1.30 & 0.55 & VLT+FORS1 & $21.20 \pm 0.01$ \\
\hline 2006 Jun 17.36557 & 2.83515 & $V$ & $4 \times 90$ & 1.14 & 0.50 & VLT+FORS1 & $22.16 \pm 0.02$ \\
\hline 2006 Jun 18.39119 & 3.86077 & V & $6 \times 180$ & 1.17 & 0.80 & VLT+FORS1 & $22.47 \pm 0.02$ \\
\hline 2006 Jul 08.33006 & 23.79964 & $V$ & $2 \times 120$ & 1.16 & 1.20 & VLT+FORS1 & $22.75 \pm 0.07$ \\
\hline $2006 \mathrm{Jul} 17.31686$ & 32.78644 & $V$ & $5 \times 120$ & 1.17 & 1.10 & VLT+FORS1 & $22.74 \pm 0.09$ \\
\hline 2006 Jun 15.12757 & 0.59715 & $R$ & $1 \times 20$ & 1.16 & 2.44 & VLT+FORS2 & $19.41 \pm 0.03$ \\
\hline 2006 Jun 15.12927 & 0.59885 & $R$ & $1 \times 60$ & 1.22 & 2.41 & $\mathrm{VLT}+\mathrm{FORS} 2$ & $19.42 \pm 0.02$ \\
\hline 2006 Jun 15.13031 & 0.59989 & $R$ & $1 \times 60$ & 1.22 & 2.39 & VLT+FORS2 & $19.41 \pm 0.02$ \\
\hline 2006 Jun 15.13136 & 0.60094 & $R$ & $1 \times 60$ & 2.44 & 2.37 & VLT+FORS2 & $19.43 \pm 0.02$ \\
\hline 2006 Jun 15.13242 & 0.60200 & $R$ & $1 \times 60$ & 2.41 & 2.35 & VLT+FORS2 & $19.43 \pm 0.02$ \\
\hline 2006 Jun 15.13355 & 0.60313 & $R$ & $1 \times 60$ & 2.39 & 2.33 & $\mathrm{VLT}+\mathrm{FORS} 2$ & $19.42 \pm 0.02$ \\
\hline 2006 Jun 15.13460 & 0.60418 & $R$ & $1 \times 60$ & 2.37 & 2.31 & VLT+FORS2 & $19.44 \pm 0.02$ \\
\hline 2006 Jun 15.13566 & 0.60524 & $R$ & $1 \times 60$ & 2.35 & 2.29 & VLT+FORS2 & $19.43 \pm 0.02$ \\
\hline 2006 Jun 15.13672 & 0.60630 & $R$ & $1 \times 60$ & 2.33 & 2.27 & $\mathrm{VLT}+\mathrm{FORS} 2$ & $19.43 \pm 0.02$ \\
\hline 2006 Jun 15.40052 & 0.87010 & $R$ & $1 \times 10$ & 2.31 & 1.16 & VLT+FORS2 & $19.83 \pm 0.03$ \\
\hline 2006 Jun 15.43038 & 0.89996 & $R$ & $1 \times 60$ & 2.27 & 1.22 & VLT+FORS2 & $19.87 \pm 0.02$ \\
\hline 2006 Jun 16.25625 & 1.72583 & $R$ & $2 \times 120$ & 1.28 & 1.22 & VLT+FORS1 & $20.85 \pm 0.01$ \\
\hline 2006 Jun 16.40016 & 1.86974 & $R$ & $2 \times 120$ & 1.17 & 0.45 & VLT+FORS1 & $20.99 \pm 0.01$ \\
\hline 2006 Jun 17.37241 & 2.84199 & $R$ & $2 \times 120$ & 1.14 & 0.50 & VLT+FORS1 & $21.76 \pm 0.02$ \\
\hline 2006 Jun 18.39941 & 3.86899 & $R$ & $6 \times 180$ & 1.19 & 0.65 & VLT+FORS1 & $22.01 \pm 0.02$ \\
\hline 2006 Jun 19.37407 & 4.84365 & $R$ & $2 \times 180$ & 1.15 & 0.50 & VLT+FORS1 & $22.18 \pm 0.04$ \\
\hline 2006 Jun 21.27125 & 6.74083 & $R$ & $3 \times 180$ & 1.20 & 0.60 & VLT+FORS1 & $22.33 \pm 0.03$ \\
\hline 2006 Jun 25.34483 & 10.81441 & $R$ & $2 \times 300$ & 1.14 & 0.90 & VLT+FORS1 & $22.44 \pm 0.03$ \\
\hline 2006 Jun 29.30301 & 14.77259 & $R$ & $11 \times 180$ & 1.16 & 0.50 & VLT+FORS1 & $22.46 \pm 0.03$ \\
\hline 2006 Jul 04.20860 & 19.67818 & $R$ & $6 \times 240$ & 1.28 & 0.60 & VLT+FORS1 & $22.46 \pm 0.04$ \\
\hline 2006 Jul 08.33536 & 23.80494 & $R$ & $2 \times 120$ & 1.16 & 1.40 & VLT+FORS1 & $22.44 \pm 0.06$ \\
\hline 2006 Jul 17.32709 & 32.79667 & $R$ & $3 \times 180$ & 1.19 & 1.10 & VLT+FORS1 & $22.51 \pm 0.08$ \\
\hline 2006 Jul 29.26643 & 44.73601 & $R$ & $5 \times 240$ & 1.15 & 0.90 & VLT+FORS1 & $22.46 \pm 0.04$ \\
\hline 2006 Aug 18.23409 & 64.70367 & $R$ & $12 \times 300$ & 1.18 & 0.60 & VLT+FORS1 & $22.44 \pm 0.04$ \\
\hline 2006 Jun 16.26278 & 1.73236 & $I$ & $3 \times 120$ & 1.26 & 0.55 & VLT+FORS1 & $20.49 \pm 0.01$ \\
\hline 2006 Jun 17.37868 & 2.84826 & $I$ & $3 \times 120$ & 1.15 & 0.43 & VLT+FORS1 & $21.25 \pm 0.02$ \\
\hline 2006 Jun 18.38882 & 3.85840 & $I$ & $4 \times 300$ & 1.16 & 0.75 & VLT+FORS1 & $21.50 \pm 0.02$ \\
\hline 2006 Jul 08.34050 & 23.81008 & $I$ & $2 \times 120$ & 1.17 & 1.10 & VLT+FORS1 & $21.70 \pm 0.06$ \\
\hline 2006 Jul 17.33614 & 32.80572 & $I$ & $3 \times 180$ & 1.20 & 0.95 & VLT+FORS1 & $21.76 \pm 0.07$ \\
\hline 2006 Jun 15.30022 & 0.76980 & $J$ & $15 \times 60$ & 1.13 & 1.20 & NTT+SofI & $18.51 \pm 0.02$ \\
\hline 2006 Jun 15.28454 & 0.75411 & $K$ & $8 \times 60$ & 1.16 & 1.15 & NTT+SofI & $17.23 \pm 0.07$ \\
\hline 2006 Jun 15.29074 & 0.76032 & $K$ & $7 \times 60$ & 1.15 & 1.15 & NTT+SofI & $17.26 \pm 0.08$ \\
\hline 2006 Jun 16.20839 & 1.67797 & $K$ & $10 \times 60$ & 1.40 & 1.10 & NTT+SofI & $18.18 \pm 0.09$ \\
\hline
\end{tabular}

